\title{
A behavioral model of unipolar resistive RAMs and its application to HSPICE integration
}

\author{
Nobuo Akou ${ }^{1}$, Tetsuya Asai ${ }^{1 a)}$, Takeshi Yanagida ${ }^{2}$, \\ Tomoji Kawai ${ }^{2}$, and Yoshihito Amemiya ${ }^{1}$ \\ ${ }^{1}$ Graduate School of Information Science and Technology, Hokkaido University \\ Kita 14, Nishi 9, Kita-ku, Sapporo 060-0814, Japan \\ 2 The Institute of Scientific and Industrial Research, Osaka University \\ 8-1 Mihogaoka, Ibaraki, Osaka 567-0047, Japan \\ a)asai@ist.hokudai.ac.jp
}

\begin{abstract}
We propose a behavioral model of unipolar resistive RAMs (ReRAMs). The model integrates three behavioral characteristics of unipolar ReRAMs; i.e., i) ON/OFF resistive switching characteristics for transient voltage pulses, ii) I-V characteristics exhibiting ON/OFF resistive switching with current compliance, and iii) currentcompliance dependence of both ON resistance and OFF threshold currents. The model is described by three nonlinear differential equations with several static functions, which enables us to employ the model in any numerical or circuit simulator. As an example, we integrate the model on HSPICE, and show the simulated results using experimental parameters extracted from fabricated ReRAMs of $\mathrm{TiO}_{2}$ thin films.
\end{abstract}

Keywords: Resistive RAM (ReRAM), behavioral model, SPICE

Classification: Integrated circuits

\section{References}

[1] A. Sawa, "Resistive switching in transition metal oxides," Materials Today, vol. 11, no. 6, pp. 28-36, June 2008.

[2] G. S. Snider, "Self-organized computation with unreliable, memristive nanodevices," Nanotechnology, vol. 18, no. 36, 365202, Dec. 2007.

[3] M. Itoh and L. O. Chua, "Memristor oscillators," Int. J. Bifurcation and Chaos, vol. 18, no. 11, pp. 3183-3206, Nov. 2008.

[4] M. Itoh and L. O. Chua, "Memristor cellular automata and memristor discrete-time cellular neural networks," Int. J. Bifurcation and Chaos, vol. 19, no. 11, pp. 3605-3656, Nov. 2009.

[5] S. H. Jo, T. Chang, I. Ebong, B. B. Bhadviya, P. Mazumder, and W. Lu, "Nanoscale memristor device as synapse in neuromorphic systems," Nano Letters, vol. 10, no. 4, pp. 1297-1301, March 2010.

[6] J.-G. Lee, D. H. Kim, J. G. Lee, D. M. Kim, and K.-S. Min, "A compact HSPICE macromodel of resistive RAM," IEICE Electron. Express, vol. 4, no. 19, pp. 600-605, Aug. 2007. 
[7] C. Rohde, B. J. Choi, D. S. Jeong, S. Choi, J.-S. Zhao, and C. S. Hwang, "Identification of a determining parameter for resistive switching of $\mathrm{TiO}_{2}$ thin films," Appl. Phys. Lett., vol. 86, no. 26, 262907, June 2005.

\section{Introduction}

Resistive RAMs (ReRAMs), where the resistance is changed by voltage and current biases, have extensively been studied to develop high-speed and largecapacity nonvolatile memories as well as functional nonvolatile memories [1]. ReRAMs are so far intended for use as alternatives to contemporary flash memories, but the applications are not limited to Boolean alternatives indeed. For example, an artificial neural computing system has been proposed where bipolar memristive characteristics of nanojunctions were fully utilized [2]. Furthermore, nonlinear oscillators have been designed by using passive or active bipolar memristors for possible applications in neuromorphic systems [3], whereas memristor cellular automata have also been proposed, aiming at parallel image-processing applications [4]. More recently, a memristive synapse, which precisely mimics spike-timing dependent plasticity of neuronal synapses, has been proposed for developing brain-like (neuromorphic) computers [5].

Although physical mechanisms of resistive switching in ReRAMs have not fully been understood, to explore novel computing architectures for ReRAMs (e.g., brain-like computers), mathematical or circuit-level ReRAM models based on behavioral characteristics of ReRAMs have to be developed in advance. Behavioral mathematical models of "bipolar" ReRAMs (memristors) have already been proposed in the literature, and have widely been accepted by many researchers (e.g., $[2,3,4,5])$. For "unipolar" ReRAMs, a behavioral circuit model has been proposed for a popular circuit simulator (HSPICE) [6]. The unipolar model showed good agreement with experimental results of transient $\mathrm{ON} / \mathrm{OFF}$ switching characteristics. Although practical unipolar ReRAMs exhibit innegligible dependence of the compliance currents on the resistance and the threshold values (e.g., [7]), the ON resistance and OFF threshold current in the unipolar model were fixed, and were independent of the compliance current. Furthermore, the unipolar model consisted of many nonlinear circuit elements; i.e., an RS flip flop for ON/OFF storage, six transfer gates for $\mathrm{ON} / \mathrm{OFF}$ switching, two operational amplifiers (comparators), etc., which may prevent us from very-large-scale circuit-level simulations. It was also very hard to transfer the model in numerical simulators (MATLAB, etc.) due to the lack of explicit definition of the model dynamics.

In this paper, we propose a behavioral mathematical model of "unipolar" ReRAMs which has compliance-current dependence of the ON resistance and OFF threshold current. The model is described by three simple dynamics which can easily be integrated in any numerical or circuit simulator. As an example, we integrate the model on HSPICE, and show the simulated results using experimental parameters extracted from fabricated ReRAMs of $\mathrm{TiO}_{2}$ 
thin films.

\section{Behavioral model of unipolar ReRAMs}

To mimic the ON/OFF storage behavior of unipolar ReRAMs, we first assume the following double-well potential function ${ }^{1}$ :

$$
H(u) \equiv \frac{1}{2} u^{2}-\beta^{-1} \ln \cosh [\beta(u-v)]
$$

where $u$ represents the $\mathrm{ON} / \mathrm{OFF}$ state $(u=1$ : $\mathrm{ON}$ and $u=-1$ : $\mathrm{OFF}), v$ the $\mathrm{ON} / \mathrm{OFF}$ transition control input, and $\beta$ the gain $(\gg 1)$. Both $u$ and $v$ are dimensionless. When $-1<v<1$, Eq. (1) forms a double well ( $H$ has two potential valleys at $u= \pm 1$ ), and the potential valley at $u=1$ (or $u=-1$ ) disappears when $v \geq 1$ (or $v \leq-1$ ). Therefore when $v \geq 1$, "OFF" state is stable, whereas "ON" state is stable when $v \leq-1$. The resistance of a ReRAM is then expressed in terms of $u$ as

$$
R_{\mathrm{ReRAM}}=\frac{1}{2}\left[\left(R_{\mathrm{on}}-R_{\mathrm{off}}\right) u+R_{\mathrm{on}}+R_{\mathrm{off}}\right]
$$

where $R_{\text {on }}$ and $R_{\text {off }}$ represent the ON and OFF resistance, respectively.

Here let us introduce the dynamics of $u$. Since the stable condition is given by $d u / d t=-\nabla H$, we obtain

$$
\tau_{1} \frac{d u}{d t}=-u+\tanh [\beta(u-v)]
$$

where $\tau_{1}$ represents the ON/OFF switching time constant. The control input $(v)$ is defined by the following differential equation:

$$
\tau_{2} \frac{d v}{d t}=-v+u \cdot \alpha(u)
$$

where $\alpha(u)$ is the static $\mathrm{ON} \rightarrow \mathrm{OFF}$ or $\mathrm{OFF} \rightarrow \mathrm{ON}$ function, and $\tau_{2}$ the fidelity time constant to $\alpha$ (one may assume $\tau_{2} \approx \tau_{1}$ ). The function $\alpha$ is defined as

$$
\alpha(u)=\frac{1}{2}\left[\left(n_{\mathrm{off}}-n_{\mathrm{on}}\right) u+n_{\mathrm{off}}+n_{\mathrm{on}}\right]
$$

where $n_{\text {off }}$ and $n_{\text {on }}$ represent normalized $\mathrm{ON} \rightarrow \mathrm{OFF}$ and $\mathrm{OFF} \rightarrow \mathrm{ON}$ conditions, respectively. The normalized conditions are given by

$$
n_{\text {off }}=\left|\frac{I_{\text {ReRAM }}}{I_{\text {th }}^{\text {off }}}\right|, \quad n_{\text {on }}=\left|\frac{V_{\text {ReRAM }}}{V_{\text {th }}^{\text {on }}}\right|
$$

where $I_{\text {ReRAM }}$ represents the current across a ReRAM, $V_{\text {ReRAM }}$ the voltage across the ReRAM, $I_{\text {th }}^{\text {off }}$ the $\mathrm{ON} \rightarrow \mathrm{OFF}$ threshold current of the ReRAM, and $V_{\mathrm{th}}^{\mathrm{on}}$ the $\mathrm{OFF} \rightarrow \mathrm{ON}$ threshold voltage of the ReRAM $(>0)$.

Now, let us see how the dynamical model works. When $u=-1$ (OFF state), $v$ approaches $-n_{\text {off }}$ because $\alpha(u)=n_{\text {off }}$, and $n_{\text {off }}$ exceeds 1 if $V_{\text {ReRAM }}$

\footnotetext{
${ }^{1}$ In principle, one may assume any double-well function here, e.g., $H(u)=-u^{2} / 2+$ $u^{4} / 4+\xi$, if the stable points can be controlled by input $\xi$. In this example, $\xi \equiv u \cdot v$ yields qualitatively the same characteristics as Eq. (1), however, the stable point is shifted by the amount of $\xi$, which results in a nonlinear form of Eqs. (2) and (5). We therefore employ the form of Eq. (1) where the stable point is $u=1$ or -1 regardless of values of $v$.
} 
exceeds the threshold $\left(V_{\mathrm{th}}^{\mathrm{on}}\right)$, which results in the disappearance of the potential barrier because $v \leq-1$. Therefore, $\mathrm{OFF} \rightarrow \mathrm{ON}$ transition happens $(u=-1 \rightarrow 1)$. On the other hand, $v$ approaches $n_{\text {on }}$ when $u=1$ (ON state), and $n_{\text {on }}$ exceeds 1 if $I_{\text {ReRAM }}$ exceeds the threshold $\left(I_{\text {th }}^{\text {off }}\right)$, which results in the $\mathrm{ON} \rightarrow \mathrm{OFF}$ transition $(u=1 \rightarrow-1)$.

The values of ON resistance $\left(R_{\mathrm{ON}}\right)$ and $\mathrm{OFF}$ threshold current $\left(I_{\mathrm{th}}^{\mathrm{off}}\right)$ depend on the value of the compliance current at the $\mathrm{OFF} \rightarrow \mathrm{ON}$ transition (e.g., [7]). To mimic this phenomenon, the compliance current has to be memorized in the model as an internal state. To this end, we introduce

$$
\tau_{3} \frac{d I_{\mathrm{comp}}^{\mathrm{OFF} \rightarrow \mathrm{ON}}}{d t}=\gamma(u) \cdot\left(-I_{\mathrm{comp}}^{\mathrm{OFF} \rightarrow \mathrm{ON}}+I_{\mathrm{ReRAM}}\right), \quad \gamma(u)=\frac{1-u}{2}
$$

where $I_{\mathrm{comp}}^{\mathrm{OFF} \rightarrow \mathrm{ON}}$ is the compliance current (to be memorized) and $\tau_{3}$ the time constant. Because $\gamma(u)$ is 0 (or 1 ) when $u=1$ (or $u=-1$ ), $I_{\mathrm{comp}}^{\mathrm{OFF} \rightarrow \mathrm{ON}}$ approaches $I_{\text {ReRAM when }} u=-1$ (OFF state), whereas $d I_{\text {comp }}^{\mathrm{OFF}} / d t$ becomes 0 when $u=1$ (ON state). Consequently, Eq. (7) integrates $I_{\text {ReRAM }}$ during the $\mathrm{OFF}$ state, and at the timing of $\mathrm{OFF} \rightarrow \mathrm{ON}$ transition, $I_{\text {ReRAM }}$, which is equal to the compliance current at the $\mathrm{ON}$ state, is preserved as $I_{\mathrm{comp}}^{\mathrm{OFF} \rightarrow \mathrm{ON}}$. Note that one has to ensure $\tau_{3} \ll \tau_{1}$ because $I_{\text {comp }}^{\mathrm{OFF} \rightarrow \mathrm{ON}}$ has to be preserved before the completion of $\mathrm{OFF} \rightarrow \mathrm{ON}$ switching transition.

Now the ON resistance and OFF threshold currents are described in terms of functions of $I_{\mathrm{comp}}^{\mathrm{OFF} \rightarrow \mathrm{ON}}$ as $R_{\mathrm{ON}}\left(I_{\mathrm{comp}}^{\mathrm{OFF} \rightarrow \mathrm{ON}}\right)$ and $I_{\mathrm{th}}^{\mathrm{off}}\left(I_{\mathrm{comp}}^{\mathrm{OFF} \rightarrow \mathrm{ON}}\right)$, respectively. The overall model ${ }^{2}$ is represented by a set of closed equations, i.e., Eqs. (2) to $(7)$ with the compliance functions $R_{\mathrm{ON}}\left(I_{\mathrm{comp}}^{\mathrm{OFF} \rightarrow \mathrm{ON}}\right)$ and $I_{\mathrm{th}}^{\text {off }}\left(I_{\mathrm{comp}}^{\mathrm{OFF} \rightarrow \mathrm{ON}}\right)$.

\section{Experimental and simulation results}

Figure 1 (a) shows our target ReRAM device. The $\mathrm{TiO}_{2}$ thin films were grown on $\mathrm{Pt} / \mathrm{Ti} / \mathrm{Si}(100)$ substrate by pulsed laser deposition method (ArF excimer, $\lambda=193 \mathrm{~nm}$ ). The film thickness was controlled to be $40 \mathrm{~nm}$. Prior to the deposition of $\mathrm{TiO}_{2}$ thin films, $\mathrm{Pt}$ (the bottom electrode) was de-
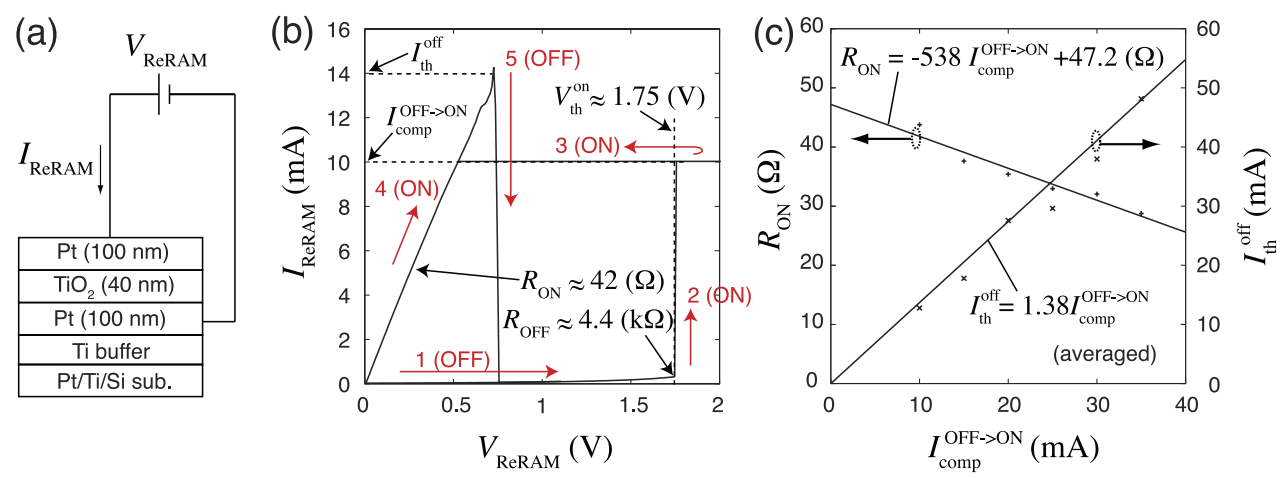

Fig. 1. Target ReRAM device and characteristics.

\footnotetext{
${ }^{2}$ The initial "forming" operation was omitted from the model for simplicity, but one may easily add an additional potential that represents the forming status, by using the same double-well strategy (formed or non-formed).
} 
posited onto the substrate with Ti buffer layer. The thickness of Pt bottom electrode was $100 \mathrm{~nm}$. The top electrode was then deposited onto the $\mathrm{TiO}_{2}$ thin films by using a metal mask with an area of $50 \times 50 \mu \mathrm{m}^{2}$. Current-voltage characteristics [ $I_{\text {ReRAM }}$ vs $V_{\text {ReRAM }}$ defined in Fig. 1 (a)] were evaluated by a semiconductor parameter analyzer (Keithley 4200-SCS) at room temperature in ambient atmosphere.

Figure 1 (b) shows the I-V characteristics. Initially, the ReRAM device was set at "off state" after the forming process. During the first forward and backward sweep of $V_{\text {ReRAM }}$ [red arrows indexed by 1, 2 (forward) and 3 (backward) in Fig. 1(b)], the parameter analyzer's compliance current was set at $10 \mathrm{~mA}$, to avoid the breakdown in the on-state device. In the first forward sweep (arrows indexed by 1 and 2), when $V_{\text {ReRAM }}$ exceeded onthreshold voltage $V_{\mathrm{th}}^{\mathrm{on}}(\approx 1.75 \mathrm{~V})$, the device was turned on. Then in the subsequent backward sweep (an arrow indexed by 3), the device was kept in the on state. Before the second forward sweep (arrows indexed by 4 and $5)$, the parameter analyzer's current compliance was disabled. In the second forward sweep, when $I_{\text {ReRAM }}$ exceeded off-threshold current $I_{\mathrm{th}}^{\text {off }}(\approx 14 \mathrm{~mA})$, the device was turned off again. These correspond to typical operations of unipolar ReRAMs [1]. Figure 1 (c) shows the $I_{\text {comp }}^{\mathrm{OFF} \rightarrow \mathrm{ON}}$ dependence of ON resistance $\left[R_{\mathrm{ON}}\left(I_{\mathrm{Comp}}^{\mathrm{OFF} \rightarrow \mathrm{ON}}\right)\right]$ and the dependence of the OFF threshold current $\left[I_{\mathrm{th}}^{\mathrm{off}}\left(I_{\mathrm{comp}}^{\mathrm{OFF} \rightarrow \mathrm{ON}}\right)\right]$, which represented the ReRAM's innegligible dependence of the compliance currents on the resistance and the threshold values. From Figs. 1 (b) and (c), we extracted the following parameter values and functions:

$$
\begin{aligned}
R_{\mathrm{OFF}} & =4.4(\mathrm{k} \Omega), \quad R_{\mathrm{ON}}\left(I_{\mathrm{comp}}^{\mathrm{OFF} \rightarrow \mathrm{ON}}\right)=-538 \cdot\left|I_{\mathrm{comp}}^{\mathrm{OFF} \rightarrow \mathrm{ON}}\right|+47.2(\Omega), \\
V_{\mathrm{th}}^{\mathrm{on}} & =1.75(\mathrm{~V}), \quad I_{\mathrm{th}}^{\mathrm{off}}\left(I_{\mathrm{comp}}^{\mathrm{OFF}}\right)=1.38 \cdot I_{\mathrm{Comp}}^{\mathrm{OFF} \rightarrow \mathrm{ON}},
\end{aligned}
$$

where $R_{\mathrm{OFF}}$ was approximately obtained by calculating differential resistance $V_{\mathrm{th}}^{\mathrm{on}} / I_{\text {ReRAM }}$ with subcritical $I_{\text {ReRAM }}(\approx 0.4 \mathrm{~mA})$ in the off state.

Figure 2 shows HSPICE netlists of the proposed ReRAM model. Figure 2 (a) represents the ReRAM subcircuit. The 1.h.s. and r.h.s. of Eqs. (3), (4) and (7) were represented by currents of capacitors (HSPICE's 'c' elements) and voltage-controlled currents ('g' elements), respectively, while the rest static functions [Eqs. (2), (5), and (6)] were implemented by 'e' elements as voltages. ${ }^{3}$ The extracted device parameters above were described in the ".param" cards. Two terminals across a ReRAM are represented by nodes "n1" and " 2 " in the figure. To ensure the timing constraints $\left(\tau_{1} \approx \tau_{2} \gg \tau_{3}\right)$, we assume $\tau_{3}=10^{-3} \tau_{1}$ and $\tau_{1}=\tau_{2}$ in the subcircuit. Figures $2(\mathrm{~b})$ and (c)

\footnotetext{
${ }^{3}$ In general, one may simulate any ordinary differential equation (ODE) on SPICE. Assume that we solve ODE $\tau_{1} d x_{1} / d t=f\left(x_{1}, x_{2}, \cdots\right)$ on SPICE. The equivalent circuit can be represented by a 'c' element (capacitor) and a ' $\mathrm{g}$ ' element (voltage-controlled current source) that accepts voltages $\left(v_{1}, v_{2}, \cdots\right)$, as shown in the inset of Fig. 2 (a). Since capacitor's current is given by $C \cdot d v_{1} / d t$, where $C$ and $v_{1}$ are the capacitance and the voltage across the capacitor, respectively, one obtains a node equation of $C \cdot d v_{1} / d t=I_{0} \cdot f\left(v_{1}, v_{2}, \cdots\right)$ where $I_{0} \cdot f(\cdot)$ represents 'g' element's current. By comparing the original equation $\left[\tau_{1} d x / d t=f\left(x_{1}, x_{2}, \cdots\right)\right]$ and this node equation, one may obtain the normalized conditions: $x_{1}=v_{1} /[1 \mathrm{~V}]$ and $\tau_{1}=C / I_{0}$. Therefore, by assuming $I_{0}=1[\mathrm{~A}]\left(\tau_{1}=C /[1 \mathrm{~A}]\right)$, all the capacitances of the 'c' elements in Fig. 2 (a) can be replaced with the time constants of Eqs. (3), (4) and (7).
} 


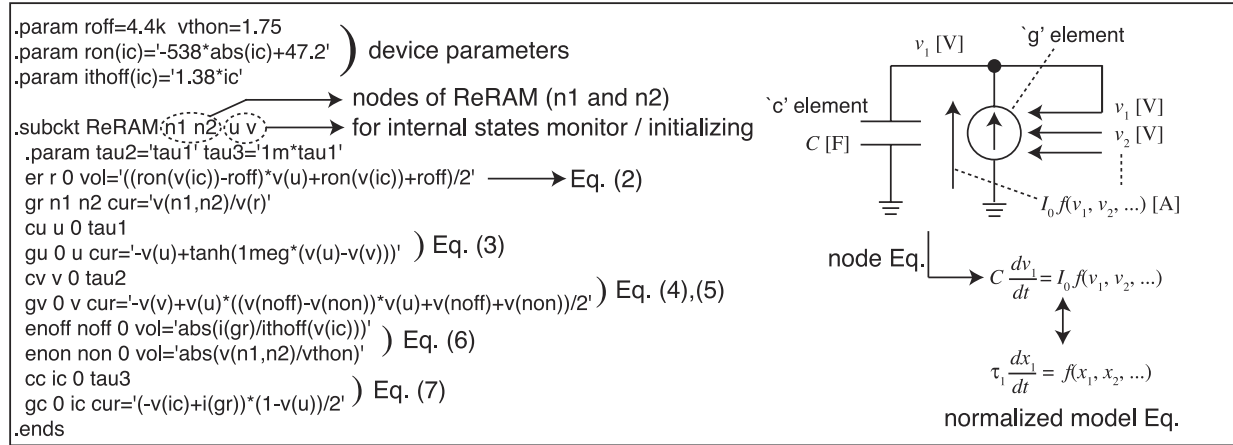

(a) ReRAM subcircuit

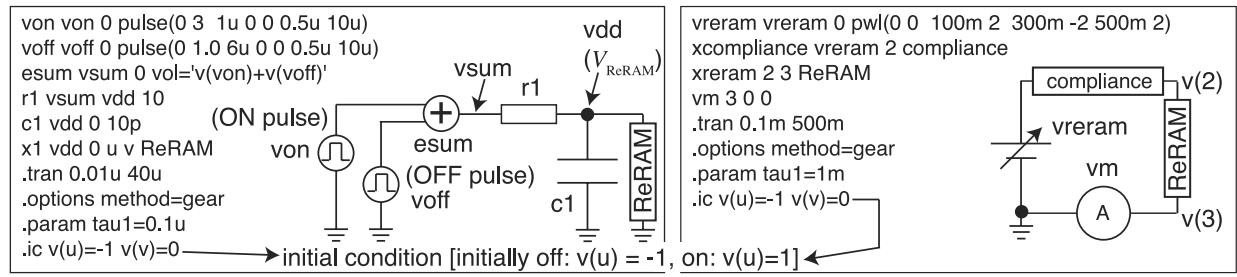

(b) Test fixture for simulating pulse responses

(c) Test fixture for simulating I-V characteristics

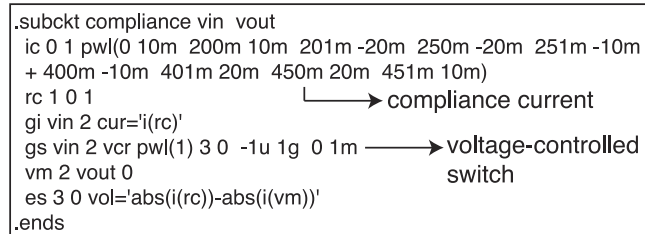

(d) Current compliance unit

Fig. 2. HSPICE netlists of behavioral ReRAM model.

show test-fixture examples for simulating pulse responses and I-V characteristics of the ReRAM model. Initial conditions of the ReRAM model (node voltages "u" and "v") are given by the ".ic" card in the test-fixture subcircuits $\left(\left.v\right|_{t=0}=0,\left.u\right|_{t=0}=-1\right.$ for initially OFF state, and $\left.u\right|_{t=0}=1$ for ON state). Measurement system's (parameter analyzer's) current compliance circuit, which is used in measuring I-V characteristics, is emulated by a subcircuit shown in Fig. 2 (d), where an external voltage source is connected to node "vin", and node "vout" is connected to the target (i.e., our ReRAM subcircuit). If the current flowing out of node "vout" exceeds a given threshold current that is defined by current source "ic" in the subcircuit, the output current is compliant to the threshold current (i.e., node voltage of "vout" is fixed over the limited states).

In the following simulations, $\beta$, initial values of $u$ and $v$ were set at $10^{6}$, -1 (OFF state) and 0 , respectively. We first demonstrate an intrinsic temporal ON/OFF switching ability of the proposed ReRAM model by applying narrow voltage pulses [frequency: $200 \mathrm{kHz}$, pulse width: $0.5 \mu \mathrm{s}$, amplitudes: $3 \mathrm{~V}$ (on pulse) and $1 \mathrm{~V}$ (off pulse), current compliance in the voltage pulser: none]. Figure 3 (a) shows the pulse responses simulated by a test fixture shown in Fig. 2 (b) $\left(\tau_{1}=100 \mathrm{~ns}\right)$. The ReRAM model was able to be turned on (or off) by applying voltage pulses of amplitude 3 (V) [or 1 (V)], as observed in typical unipolar ReRAMs [1].

Figure 3 (b) plots transient responses of the ReRAM model for transient sweep of $V_{\text {ReRAM }}\left(I_{\mathrm{c}}\right.$ in the figure) simulated by a test fixture with a current- 


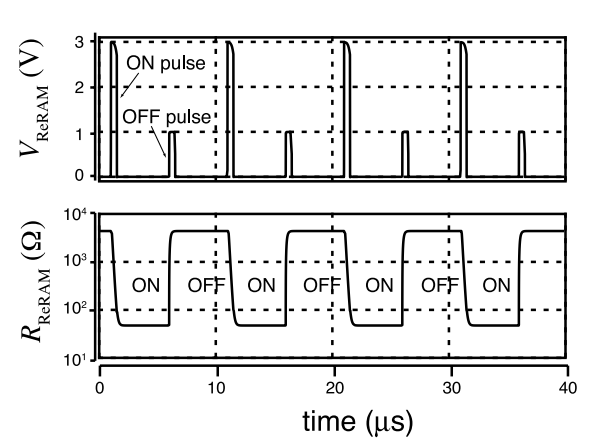

(a) pulse responses

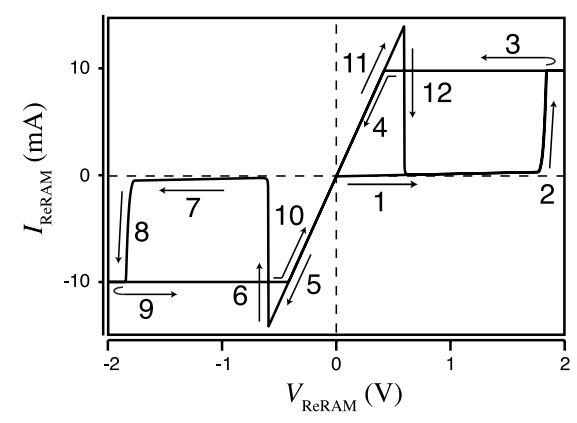

(c) I-V characteristics

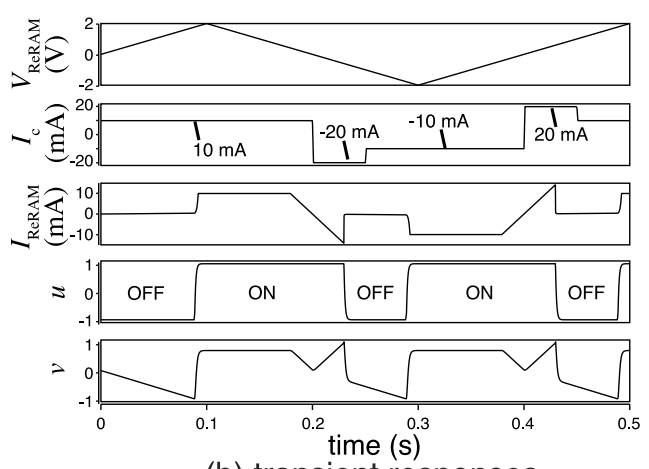

(b) transient responses
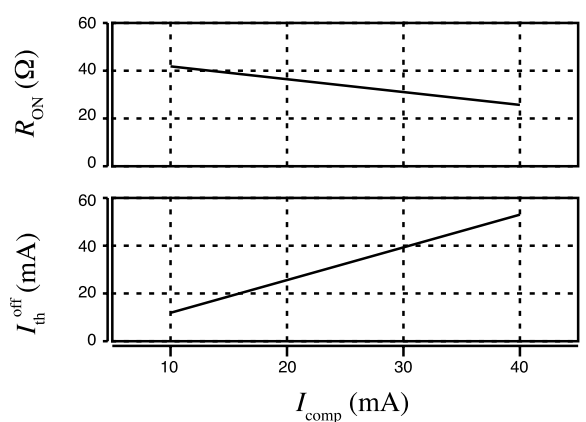

(d) current compliance dependence

Fig. 3. Simulated results (HSPICE).

compliance unit shown in Figs. $2(\mathrm{c})$ and (d). Here $\tau_{1}$ was set at $1 \mathrm{~ms}$ for fast simulation. The compliance current was essentially set at $\pm 10 \mathrm{~mA}$, but was set at $\pm 20 \mathrm{~mA}$ during the expected $\mathrm{ON} \rightarrow \mathrm{OFF}$ transition $\left[I_{\text {ReRAM }}\right.$ does not exceed this value $(20 \mathrm{~mA})$, which meant that the current compliance was disabled during the term]. The $I_{\text {ReRAM }}-V_{\text {ReRAM }}$ characteristic extracted from Fig. 3 (b) is plotted in Fig. 3 (c). Finally, the dependence of the model's ON resistance and $\mathrm{OFF}$ threshold current on the compliance current is presented in Fig. $3(\mathrm{~d})$.

\section{Conclusion}

We proposed a behavioral model of a resistive RAM (ReRAM) device having a dependence of ON resistance and OFF threshold current on values of compliance current. The model was described by a set of nonlinear differential equations which could be integrated on any numerical or circuit simulator. As an example, we integrated the model on HSPICE, and showed the typical characteristics. The results agreed well with experimental results of fabricated ReRAMs of $\mathrm{TiO}_{2}$ thin films.

\section{Acknowledgments}

This study was supported by a Grant-in-Aid for Scientific Research on Innovative Areas [20111004] from the Ministry of Education, Culture Sports, Science and Technology (MEXT) of Japan. 